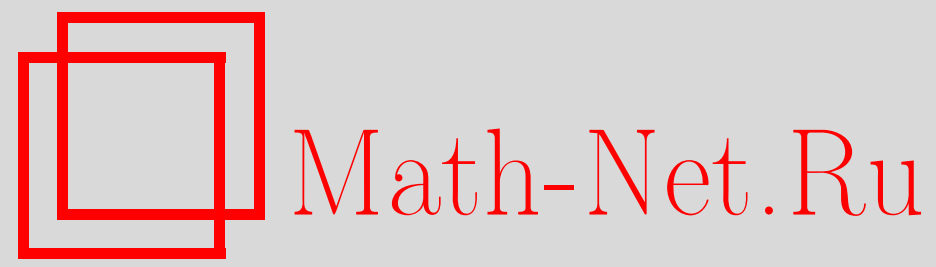

M. Broniatowski, M. Weber, Strong laws for sums of extreme values, Теория вероятн. и ее примен., 1997, том 42, выпуск $3,553-563$

DOI: https://doi.org/10.4213/tvp1952

Использование Общероссийского математического портала Math-Net.Ru подразумевает, что вы прочитали и согласны с пользовательским соглашением http://www.mathnet.ru/rus/agreement

Параметры загрузки:

IP : 3.85 .183 .62

26 апреля 2023 г., 08:55:53

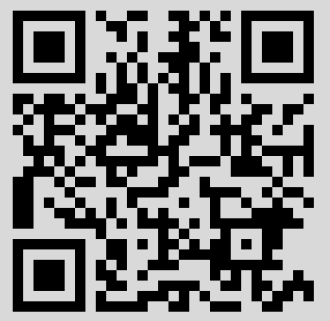




\section{STRONG LAWS FOR SUMS OF EXTREME VALUES}

В статье представлен новый подход к усиленным законам больших чисел для сумм экстремальных значений последовательностей независимых, но не обязательно одинаково распределенных случайных величин. Наш метод основан на простом распространении одного неравенства из работы [7] (M. Marcus, G. Pisier) на «хвостовые» распределения слабых $l_{p}$-норм независимых последовательностей вещественных случайных величин. Наши результаты связаны также с некоторыми обобщениями закона больших чисел МарцинкевичаЗигмунда.

Ключевые слова и Фразы: порядковые статистики, экстремальные значения, законы больших чисел, закон больших чисел Марцинкевича-Зигмунда, банаховы пространства.

\section{Introduction and notation}

Let $\left(X_{1}, \ldots, X_{n}\right)$ be strictly positive independent unbounded random variables, let $X_{1}^{(n)} \geqslant \cdots \geqslant X_{n}^{(n)}$ denote the rearrangement of $\left(X_{1}, \ldots, X_{n}\right)$ in the decreasing order. We state strong laws of large numbers for the sum of extreme values

$$
W_{n}:=\sum_{i=1}^{k_{n}} X_{i}^{(n)}
$$

where $\left(k_{n}, n \geqslant 1\right)$ is a sequence of positive integers satisfying.

(K1) $\left(k_{n}, n \geqslant 1\right)$ is non-decreasing, $1 \leqslant k_{n} \leqslant n, k_{n} \rightarrow \infty$ as $n \rightarrow \infty$ and the $X_{i}$ 's satisfy some common condition pertaining to their distribution functions (d.f's).

Mason [8] proved that, whenever the $X_{i}$ 's are independent identically distributed random variables (i.i.d. r.v's) with common d.f. $F$,

(I) A necessary and sufficient condition for

$$
T_{n}:=\frac{1}{k_{n}} \sum_{i=1}^{k_{n}} X_{i}^{(n)}-X_{k_{n}}^{(n)} \longrightarrow c \quad \text { a.s. as } n \rightarrow \infty
$$

${ }^{*}$ CNRS and Université de Reims, 57bis Rue Taittinger, 51096 Reims, France.

${ }^{* *}$ CNRS Université de Strasbourg. 
for some $c>0$ and all sequence $k_{n}=\left[n^{\alpha}\right], 0<\alpha<1$, is

$$
F \in \mathcal{E}_{c}:=\left\{F: \lim _{x \rightarrow \infty} \frac{\int_{x}^{\infty}(1-F)(y) d y}{(1-F)(x)}=c\right\}
$$

and

(II) A necessary and sufficient condition for

$$
S_{n}:=\frac{1}{k_{n}} \sum_{i=1}^{k_{n}} \log X_{i}^{(n)}-\log X_{k_{n}}^{(n)} \longrightarrow c \quad \text { a.s. as } n \rightarrow \infty
$$

for some $c>0$ and all sequence $k_{n}=\left[n^{\alpha}\right], 0<\alpha<1$, is

$$
F \in \mathbf{R}_{c}:=\left\{F: \text { for all } t>0, \lim _{x \rightarrow \infty} \frac{1-F(t x)}{1-F(x)}=t^{-1 / c}\right\} .
$$

(I) describes d.f's close to the exponential distribution, while (II) refers to regular variation. Related works, including laws of the iterated logarithm and weak convergence as well as statistical applications have been developped by Csörgö and Mason [3], Deheuvels, Haeusler and Mason [4], Deheuvels and Mason [5], Einmahl, Haeusler and Mason [6], among others.

In this paper we obtain a.s. laws for the cruder statistics $W_{n}$, under weaker assumptions than above. We impose some uniformity tail condition on the family of the d.f's $F_{i}$ of $X_{i}$. Those conditions are less restrictive than $\mathcal{E}_{c}$ and $\mathbf{R}_{c}$, since they mainly characterize moment properties. The technique used here presents some nice properties with respect to these hypotheses, as will be observed in conjunction with the Marcinkiewicz-Zygmund law of large numbers.

We now state some definition and notation.

Throughout this paper, we denote $\left(X_{n}\right)=\left(X_{1}, \ldots, X_{n}\right)$.

Let $\varphi: \mathbf{R}^{+} \rightarrow \mathbf{R}^{+}$be a continuous increasing unbounded function such that $\varphi(0)=0$, and let

$$
\begin{aligned}
\left\|\left(X_{n}\right)\right\|_{\varphi, \infty} & =\varphi^{-1}\left(\sup _{u>0}\left(\varphi(u) \operatorname{card}\left\{i \leqslant n: X_{i}>u\right\}\right)\right) \\
& =\varphi^{-1}\left(\sup _{1 \leqslant k \leqslant n} k \varphi\left(X_{k}^{(n)}\right)\right) .
\end{aligned}
$$

Whenever $\varphi(t)=t^{p}, p>1$, and when dealing with infinite sequences, it is well known that $\|\cdot\|_{\varphi, \infty}$ is equivalent to a norm on the space $l_{p, \infty}$ of all sequences $\left(x_{n}, n \geqslant 1\right)$ of real and complex numbers such that

$$
\left\|\left(x_{n}\right)_{n=1}^{\infty}\right\|_{p, \infty}=\left(\sup \left(t^{p} \operatorname{card}\left\{n:\left|x_{n}\right|>t\right\}\right)\right)^{1 / p}<\infty .
$$

Moreover, in this case $l_{p, \infty}$ is a Banach space. 
Define

$$
H_{\varphi}^{X}(n):=\sup _{u>0} \varphi(u) \sum_{i=1}^{n} \mathbf{P}\left\{X_{i}>u\right\} .
$$

The main tool used in this paper is an extension of an inequality due to Marcus and Pisier [7]. We state it in the following

Proposition 1.1. Let $\varphi: \mathbf{R}^{+} \rightarrow \mathbf{R}^{+}$be a continuous increasing unbounded function such that $\varphi(0)=0$. Let $\left(X_{1}, \ldots, X_{n}\right)$ be non-negative independent r.v's. Then

(i)

$$
\sup _{b \geqslant 0} \varphi(b) \mathbf{P}\left\{\left\|\left(X_{n}\right)\right\|_{\varphi, \infty}>b\right\} \leqslant 262 H_{\varphi}^{X}(n) .
$$

(ii) Assume that $A=\sup _{t \geqslant 0} \varphi(t) \sum_{i=1}^{n} \mathbf{P}\left\{X_{i}>t\right\}$ is finite. If there exists $0<\lambda_{0}<\infty$, such that, for all $1 \leqslant i \leqslant n, X_{i} \leqslant \varphi^{-1}\left(\lambda_{0} A\right)$ a.s., then, for all $\lambda \in[0,1]$,

$$
\mathbf{P}\left\{\left\|\left(X_{n}\right)\right\|_{\varphi, \infty}>\varphi^{-1}(\lambda A)\right\} \geqslant \frac{(1-\lambda)^{2}}{128 \lambda_{0}+2 / A} .
$$

The first part of Proposition 1.1 is a consequence of Theorem 3.3 in [7], which is applied with $p=1$ to the r.v's $Z_{i}=\varphi\left(X_{i}\right)$; see also [9] for this inequality. The second part of Proposition 1.1 is new and is proved in Section 3. Although we will not make use of it in the sequel, we find most useful to state it together with (i).

Two classes of functions $\varphi$ will be considered.

$\mathrm{C}$ a s e 1: Exponential type.

$$
\text { For any } u, v \geqslant 0, \quad \varphi(u+v) \leqslant \varphi(u) \varphi(v) .
$$

C a s e 2: Power type.

$$
\text { For any } \quad \alpha, \beta \geqslant 0, \quad \varphi(\alpha) \varphi(\beta) \leqslant \varphi(\alpha \beta) \text {. }
$$

In the i.i.d. case it will be convenient to introduce the following classes of d.f's.

Let

$$
E_{c}:=\left\{F: \lim _{x \rightarrow \infty} \frac{-\log (1-F)(x)}{x}=c\right\}
$$

and

$$
P_{c}:=\left\{F: \sup _{x \geqslant 0} x^{1 / c}(1-F)(x)<\infty\right\} .
$$

Strong laws for $W_{n}$ in the case when $F \in P_{c}$ will be handled with $\varphi(x)=x^{1 / c}$ while $\varphi(x)=\exp (x / c)-1$ will be used when $F \in E_{c}$, together with Proposition 1.1.

In order to clarify the definition of $E_{c}$ and $P_{c}$ we state two lemmas; denote by $D(\Lambda)$ the domain of attraction of the Gumbel extreme value distribution; see [1, Ch. 8]. 
Lemma 1.1. (i) If $F \in \mathcal{E}_{c}$, then $F \in E_{1 / c}$.

(ii) If $F \in E_{1 / c}$, i.e.,

$$
-\log (1-F)(x)=\frac{x}{c}(1+\delta(x))
$$

with $\lim _{x \rightarrow \infty} \delta(x)=0$, then $F \in D(\Lambda)$. Moreover, if $\lim _{x \rightarrow \infty} x \delta(x)=0$ then $F \in \mathcal{E}_{c}$.

P r o of. (i) If $F \in \mathcal{E}_{c}$, then

$$
\frac{\log \int_{x}^{\infty}(1-F)(y) d y}{\log (1-F)(x)} \longrightarrow 1 \quad \text { as } \quad x \rightarrow \infty
$$

Furthermore, for any $x>x_{0}>0$,

$$
\begin{gathered}
\log \int_{x}^{\infty}(1-F)(y) d y-\log \int_{x_{0}}^{\infty}(1-F)(y) d y \\
=-\frac{1}{c}\left(x-x_{0}\right)+\int_{x_{0}}^{x} \varepsilon(u) d u,
\end{gathered}
$$

where $\varepsilon(x) \rightarrow 0$ as $x \rightarrow \infty$.

By (1.8) and (1.9), $F \in E_{1 / c}$.

(ii) That $F \in D(\Lambda)$ whenever $\lim _{x \rightarrow \infty} \delta(x)=0$ is readily checked evaluating $\lim _{n \rightarrow \infty} F^{n}(x+c \log n)$.

Let

$$
\begin{aligned}
R(x) & :=\frac{\int_{x}^{\infty}(1-F)(y) d y}{(1-F)(x)}, \quad U(s):=\sup \{x: 1-F(x) \geqslant s\}, \\
h(x) & :=U\left(\frac{(1-F)(x)}{e}\right)-x .
\end{aligned}
$$

Since $F \in D(\Lambda), R(x)=h(x)(1+o(1))$ as $x \rightarrow \infty$. We thus prove that $\lim _{x \rightarrow \infty} h(x)=c$.

Since $F \in D(\Lambda)$,

$$
\lim _{t \rightarrow 0} \frac{U(t u)-U(t)}{U(t v)-U(t)}=\frac{\log u}{\log v} \quad \text { for all } \quad u, v>0, v \neq 1 .
$$

Set $t=(1-F)(x), u=e, v=1 / e$ in $(1.10)$ to obtain

$$
\frac{U((1-F)(x) e)-x}{h(x)} \rightarrow 1 \quad \text { as } \quad x \rightarrow \infty
$$

Since $F \in E_{1 / c}$, there exist two positive functions $\delta_{1}(x), \delta_{2}(x)$ which tend to 0 as $x \rightarrow \infty$ such that

$$
\frac{1}{c} x\left(1+\delta_{1}(x)\right) \leqslant-\log (1-F)(x) \leqslant \frac{1}{c} x\left(1+\delta_{2}(x)\right) .
$$


Using (1.11) and (1.12) together with $x \delta(x) \rightarrow 0$ as $x \rightarrow \infty$ implies that $F \in \mathcal{E}_{1 / c}$.

Lemma 1.2. If $F \in \mathbf{R}_{c}$, then $F \in P_{c^{\prime}}$ for all $c^{\prime}>c$.

$\mathrm{Pr}$ o o f. It is a consequence of Proposition 1.3.6 in [1].

The rest of this paper is organized as follows: our results are stated in Section 2; Section 3 contains the proofs.

\section{Results}

Let $X:=\left(X_{i}, 1 \leqslant i \leqslant n\right)$ be defined as in Section 1 .

Let, for any integer $p \geqslant 1$,

$$
n_{p}:=\left[\exp \left(\frac{p}{\log p}\right)\right] \text {. }
$$

Introduce sequences of integers $\left(k_{n}, n \geqslant 1\right)$ satisfying

$$
\limsup _{p \rightarrow \infty} \frac{k_{n_{p+1}}}{k_{n_{p}}}=1
$$

Case 1 (Exponential type).

Theorem 2.1. Assume that $\varphi$ satisfies (1.5) and that $H_{\varphi}^{X}(n)$ is finite for all $n \geqslant 1$. Then, for any $\alpha>1$, for any sequence $\left(k_{n}, n \geqslant 1\right)$ satisfying (K1) and (K2), we have

$$
\limsup _{n \rightarrow \infty} \frac{\sum_{i=1}^{k_{n}} X_{i}^{(n)}+\varphi^{-1}(i)}{k_{n} \varphi^{-1}\left((\log n)^{\alpha} H_{\varphi}^{X}([n \alpha])\right)} \leqslant 1 \quad \text { a.s. }
$$

In the special case where the $X_{i}$ 's are i.i.d. and if

$$
0<c:=\sup \left\{d: \mathbf{E} e^{d X_{1}}<\infty\right\},
$$

then (2.2) is replaced by

$$
\limsup _{n \rightarrow \infty} \frac{c \sum_{i=1}^{k_{n}} X_{i}^{(n)}+\log k_{n} !}{k_{n} \log n} \leqslant 1 \quad \text { a.s. }
$$

We can improve Theorem 2.1 in the i.i.d. case when some additional information on $F$ is available:

Theorem 2.2. Let $X_{1}, \ldots, X_{n}$, be non-negative i.i.d. r.v's such that $F \in E_{c}$. Then for any increasing sequence of positive numbers $k_{n}$ satisfying (K1) and (K2), together with

$$
\text { for all } \varepsilon>0, \quad k_{n}=o\left(n(\log n)^{-\varepsilon}\right) \quad \text { as } n \rightarrow \infty,
$$

we have

$$
\limsup _{n \rightarrow \infty} \frac{\sum_{i=1}^{k_{n}} X_{i}^{(n)}}{k_{n} \log \left(n / k_{n}\right)}=\frac{1}{c} \quad \text { a.s. }
$$


$\mathrm{R}$ e $\mathrm{m}$ a $\mathrm{r} \mathrm{k}$ 2.1. If, instead of $F \in E_{c}, c>0$, we only have

$$
\liminf _{x \rightarrow \infty}-\frac{\log (1-F)(x)}{x} \leqslant c
$$

then the expression in (2.6) is greater than $1 / c$ a.s.

In the same way we have

Theorem 2.3. Let $X_{1}, \ldots, X_{n}$, be non-negative i.i.d. r.v's with d.f. $F$ satisfying

$$
\liminf _{x \rightarrow \infty}-\frac{\log (1-F)(x)}{x} \leqslant c .
$$

For any sequence $k_{n}$ satisfying (K1) and (K2) together with

$$
\liminf _{n \rightarrow \infty} \frac{k_{n}}{\log \log n} \geqslant 1
$$

we have

$$
\liminf _{n \rightarrow \infty} \frac{\sum_{i=1}^{k_{n}} X_{i}^{(n)}}{k_{n} \log \left(n / k_{n}\right)} \geqslant \frac{1}{c} \quad \text { a.s. }
$$

Moreover, if $F \in E_{c}$, then

$$
\lim _{n \rightarrow \infty} \frac{\sum_{i=1}^{k_{n}} X_{i}^{(n)}}{k_{n} \log \left(n / k_{n}\right)}=\frac{1}{c} \quad \text { a.s. }
$$

for all sequence $k_{n}$ satisfying (K1), (K2), (2.5) and (2.8).

$\mathrm{R} \mathrm{e} \mathrm{m} \mathrm{a} \mathrm{r} \mathrm{k} 2.2$. Theorem 2.3 is of interest for statistical applications. Let $\left(X_{i}, 1 \leqslant i \leqslant n\right)$ be a sample of independent non-negative r.v's and let $\left(1-F_{i}\right)(x)=x^{-\alpha} l_{i}(x), \alpha>0$, where the $l_{i}$ 's are slowly varying at infinity. Let further $Y_{i}=\log ^{+} X_{i}$, where $\log ^{+} x:=\log (x \vee e)$. It can easily be verified that Theorem 2.3 holds for the r.v's $Y_{i}$. Therefore a strongly consistent estimate of the Pareto tail index $\alpha$ is

$$
\alpha_{n}=\frac{1}{k_{n} \log \left(n / k_{n}\right)} \sum_{i=1}^{k_{n}} \log ^{+} X_{i}^{(n)},
$$

where $k_{n}$ satisfies (K1), (K2), (2.5) and (2.8).

In the case where the variables $X_{i}$ are i.i.d. with common exponential distribution with mean $1 / c,(2.10)$ can be deduced from [5], Theorem 2 and 3 , where a.s. second order asymptotics for sums of extreme values are obtained through the theory of extreme values of uniform samples.

Case 2 (Power type). For any $n \geqslant 1$ let $\Delta_{\varphi}(n):=\sum_{i=1}^{n} \varphi^{-1}(1 / i)$. We state the following result.

Theorem 2.4. Assume that $\varphi$ satisfies (1.6), and that $H_{\varphi}^{X}(n)$ is finite for any $n \geqslant 1$. Then for all sequence $k_{n}$ satisfying (K1) and (K2), for any 
$\alpha>1$, we have

$$
\lim _{n \rightarrow \infty} \frac{\sum_{i=1}^{k_{n}} X_{i}^{(n)}}{\varphi^{-1}\left((\log n)^{\alpha} H_{\varphi}^{X}([n \alpha])\right) \Delta_{\varphi}\left(\left[k_{n} \alpha\right]\right)}=0 \quad \text { a.s. }
$$

Corollary 2.1. Let $X_{1}, \ldots, X_{n}$, be non-negative i.i.d. r.v's such that $F \in P_{1 / r}$ for some $r>0$. For any $b>1 / r$ we have

$$
\lim _{n \rightarrow \infty} \frac{\sum_{i=1}^{k_{n}} X_{i}^{(n)}}{n^{1 / r}(\log n)^{b}\left(\sum_{i=1}^{n} i^{-1 / r}\right)}=0 \quad \text { a.s. }
$$

$\mathrm{R} \mathrm{e} \mathrm{m}$ a $\mathrm{rk} 2.3$. This latter result is of interest when $0<r<1$. It follows readily from Theorem 2.4 , with $\varphi(u)=u^{r}$ and $k_{n}=n$. It is an extension of the Marcinkiewicz-Zygmund law of large numbers under condition $P_{1 / r}$, which is weaker than $\mathbf{E} X^{r}<\infty$; see e.g. [2] for the Marcinkiewicz-Zygmund law.

\section{Proofs}

Pro of of Proposition 1.1. As noted above there is nothing to prove concerning part (i), since it is a plain application of Theorem 3.3 of Marcus and Pisier [7] with $p=1$ to the r.v's $Z_{i}=\varphi\left(X_{i}\right)$. Consequently we prove (ii). It is enough to prove (1.4) with $\varphi(x)=x$. The general case will follow applying (1.4) to the r.v's $Z_{i}=\varphi\left(X_{i}\right)$. Let $\left(\varepsilon_{i}, 1 \leqslant i \leqslant n\right)$ be a sample of Rademacher r.v's, independent of the $X_{i}$ 's. By recentering and following closely the lines of (3.9)-(3.11) in the proof of Theorem 3.3 in [7] we obtain

$$
\mathbf{E}\left(\left\|\left(X_{n}\right)\right\|_{1, \infty}^{2}\right) \leqslant 2 A+8 \mathbf{E}\left(\sup _{t \geqslant 0}\left[t \sum_{i=1}^{n} \varepsilon_{i} 1_{\left\{X_{i}>t\right\}}\right]\right)^{2} .
$$

Further from Lemma 3.4 of [7], we have

$$
\mathbf{E}\left(\sup _{t \geqslant 0}\left[t \sum_{i=1}^{n} \varepsilon_{i} 1_{\left\{X_{i}>t\right\}}\right]\right)^{2} \leqslant 8 \sum_{i=1}^{n} \mathbf{E} X_{i}^{2}
$$

Note that

$$
\sum_{i=1}^{n} \mathbf{E} X_{i}^{2}=\int_{0}^{\lambda_{0} A} 2 t\left\{\sum_{i=1}^{n} \mathbf{P}\left(X_{i}>t\right)\right\} d t \leqslant 2 \lambda_{0} A^{2}
$$

Hence

$$
\mathbf{E}\left(\left\|\left(X_{n}\right)\right\|_{1, \infty}^{2}\right) \leqslant 2 A+128 \lambda_{0} A^{2}
$$

Further we have

$$
\mathrm{E}\left(\left\|\left(X_{n}\right)\right\|_{1, \infty}\right) \geqslant A
$$


By the Paley-Zygmund inequality for non-negative integrable random variables we have, for all $0<\lambda<1$,

$$
\mathbf{P}\{X>\lambda \mathbf{E} X\} \geqslant \frac{(1-\lambda)^{2}(\mathbf{E} X)^{2}}{\mathbf{E} X^{2}} .
$$

Combining (3.5) together with (3.3) and (3.4) we obtain that for all $0<\lambda<1$,

$$
\mathbf{P}\left\{\left\|\left(X_{n}\right)\right\|_{1, \infty}>\lambda A\right\} \geqslant \frac{(1-\lambda)^{2} A^{2}}{2 A+128 \lambda_{0} A^{2}} .
$$

This completes the proof of Proposition 1.1.

\section{Proofs concerning case 1.}

P r o of of T h e or e m 2.1. From (1.5) we have $\varphi^{-1}(\alpha)+\varphi^{-1}(\beta) \leqslant$ $\varphi^{-1}(\alpha \beta)$ for all $\alpha$ and $\beta>0$. Thus for any integer $n \geqslant 1$ and for any $i \leqslant n$, we have $X_{i}^{(n)} \leqslant \varphi^{-1}\left(\varphi\left(X_{i}^{(n)}\right) i\right)-\varphi^{-1}(i)$. Hence for all $n \geqslant 1$ and $i \leqslant n$

$$
X_{i}^{(n)}+\varphi^{-1}(i) \leqslant\left\|\left(X_{n}\right)\right\|_{\varphi, \infty} .
$$

Consequently, for any $1 \leqslant k \leqslant n$ and any $\tau>0$, we have

$$
\mathbf{P}\left\{\sum_{i=1}^{k} X_{i}^{(n)}+\varphi^{-1}(i)>\tau\right\} \leqslant \mathbf{P}\left\{\left\|\left(X_{n}\right)\right\|_{\varphi, \infty}>\frac{\tau}{k}\right\} \leqslant \frac{262 H_{\varphi}^{X}(n)}{\varphi(\tau / k)} .
$$

The last inequality follows from (1.3).

Set $\tau=k \varphi^{-1}\left(\varphi(s) H_{\varphi}^{X}(n)\right)$ with $s>0$ arbitrary in (3.8) to obtain

$$
\mathbf{P}\left\{\sum_{i=1}^{k} X_{i}^{(n)}+\varphi^{-1}(i)>k \varphi^{-1}\left(\varphi(s) H_{\varphi}^{X}(n)\right)\right\} \leqslant \frac{262}{\varphi(s)} .
$$

Writing (3.9) with $n=n_{p}, k=k_{n_{p}}, s=\varphi^{-1}\left(p^{\alpha}\right), \alpha>1$, and using Borel Cantelli lemma yields

$$
\sum_{i=1}^{k_{n_{p}}} X_{i}^{\left(n_{p}\right)}+\varphi^{-1}(i) \leqslant k_{n_{p}} \varphi^{-1}\left(p^{\alpha} H_{\varphi}^{X}\left(n_{p}\right)\right)
$$

almost surely for all $p$ large enough.

Let now $n \in\left[n_{p}, n_{p+1}\right]$. Let $\alpha^{\prime}>\alpha$. Assuming $n$ large enough we have for any $\varepsilon>0$,

$$
\begin{aligned}
\sum_{i=1}^{k_{n_{p}}}\left(X_{i}^{\left(n_{p}\right)}+\varphi^{-1}(i)\right) & \leqslant \sum_{i=1}^{k_{n_{p+1}}}\left(X_{i}^{\left(n_{p+1}\right)}+\varphi^{-1}(i)\right) \\
& \leqslant(1+\varepsilon) k_{n} \varphi^{-1}\left((\log n)^{\alpha^{\prime}} H_{\varphi}^{X}\left(\left[n \alpha^{\prime}\right]\right)\right) .
\end{aligned}
$$

This proves (2.2). 
P r o of of $\mathrm{Th}$ e o r e $\mathrm{m}$ 2.2. Since the upper inequality in (2.6) is a simple consequence of Theorem 2.1 , we just have to show that the lower bound in (2.6) holds.

Note that for any $0<\varepsilon<1$,

$$
\begin{aligned}
& \mathbf{P}\left\{X_{k}^{(n)} \leqslant \frac{\varepsilon}{c} \log \frac{n}{k}\right\} \\
& \quad \leqslant \mathbf{P}\left\{\exists I \subset\{1, \ldots, n\}: \operatorname{card} I=n-k, \sup _{i \in I} X_{i} \leqslant \frac{\varepsilon}{c} \log \frac{n}{k}\right\} .
\end{aligned}
$$

Note further that when $I \subset\{1, \ldots, n\}$,

$$
\mathbf{P}\left\{\sup _{i \in I} X_{i} \leqslant \frac{\varepsilon}{c} \log \frac{n}{k}\right\}=\exp \left(-(n-k) \mathbf{P}\left\{X_{1}>\frac{\varepsilon}{c} \log \frac{n}{k}\right\}\right)(1+o(1))
$$

as $n / k \rightarrow \infty$.

Let $\beta>1$ such that $\gamma=\varepsilon \beta<1$. By assumption $E_{c}$, when $n / k$ is large,

$$
\mathbf{P}\left\{\sup _{i \in I} X_{i} \leqslant \frac{\varepsilon}{c} \log \frac{n}{k}\right\}=\exp \left(-(1-\varepsilon) k^{\gamma} n^{1-\gamma}\right)(1+o(1)) \text {. }
$$

For $n$ large enough we thus have

$$
\mathbf{P}\left\{X_{k}^{(n)} \leqslant \frac{\varepsilon}{c} \log \frac{n}{k}\right\} \leqslant n^{k} \exp \left(-(1-\varepsilon) k^{\gamma} n^{1-\gamma}\right) .
$$

We now choose $k \leqslant n / 2(\log n)^{1-\gamma}$. This is precisely what is assumed in (2.5) when $k=k_{n}$. Consequently, for $n$ large we have

$$
\mathbf{P}\left\{X_{k_{n}}^{(n)} \leqslant \frac{\varepsilon}{c} \log \frac{n}{k_{n}}\right\} \leqslant \exp \left(-\frac{1}{2} k_{n} \log n\right)
$$

which tends to 0 as $n \rightarrow \infty$. By Borel Cantelli lemma and letting $\varepsilon$ tend to 1 , we obtain (2.6).

P r o of of $\mathrm{Th}$ e ore $\mathrm{m}$ 2.3. Let $0<\delta<1$ and $0<\varepsilon<1$. For any $n \geqslant 1$, set

$$
\alpha_{n}=\frac{1}{c(1-\delta)(1+\varepsilon)} k_{n} \log \frac{n}{k_{n}}
$$

where the sequence $k_{n}$ satisfies (K1) and (K2).

In a first step we prove

$$
\sum_{p \geqslant 1} \mathbf{P}\left\{\sum_{i=1}^{k_{n_{p}}} X_{i}^{\left(n_{p}\right)} \leqslant \alpha_{n_{p}}\right\}<\infty
$$

In order to derive (3.14), observe that

$$
\mathbf{P}\left\{\sum_{i=1}^{k_{n}} X_{k}^{(n)} \leqslant(1-\delta) \alpha_{n}\right\} \leqslant \mathbf{P}\left\{X_{k_{n}}^{(n)} \leqslant \frac{(1-\delta) \alpha_{n}}{n}\right\}
$$


Using the same argument as along lines (3.11)-(3.12) we have

$$
\mathbf{P}\left\{X_{k_{n}}^{(n)} \leqslant \frac{(1-\delta) \alpha_{n}}{n}\right\} \leqslant \exp \left(-\left(n-k_{n}\right) \mathbf{P}\left\{X_{1}>\frac{(1-\delta) \alpha_{n}}{n}\right\}\right)
$$

for $n$ large. The last inequality follows from (2.7). We thus obtain

$$
\mathbf{P}\left\{\sum_{i=1}^{k_{n_{p}}} X_{k}^{\left(n_{p}\right)} \leqslant(1-\delta) \alpha_{n_{p}}\right\} \leqslant \frac{1}{p^{1+\gamma}},
$$

for some $\gamma>0$, assuming $p$ large.

Hence (3.14) holds. The proof of Theorem 2.3 is achieved by using similar arguments as in the end of the proof of Theorem 2.2. The second part of the statement is obtained combining Theorem 2.2 together with (2.9).

\section{Proofs concerning case 2.}

Th e $\mathrm{P}$ r o of of $\mathrm{T}$ h e o r e m 2.4 is similar to that of Theorem 2.1.

From (1.6) it follows that for all $1 \leqslant k \leqslant n$,

$$
\begin{aligned}
X_{k}^{(n)} & =\varphi^{-1}\left(\varphi\left(X_{k}^{(n)} \frac{k}{k}\right)\right) \leqslant \varphi^{-1}\left(\varphi\left(X_{k}^{(n)} k\right)\right) \varphi^{-1}\left(\frac{1}{k}\right) \\
& \leqslant \varphi^{-1}\left(\frac{1}{k}\right)\left\|\left(X_{n}\right)\right\|_{\varphi, \infty} .
\end{aligned}
$$

Therefore,

$$
\sum_{i=1}^{k_{n}} X_{i}^{(n)} \leqslant\left\|\left(X_{n}\right)\right\|_{\varphi, \infty} \sum_{i=1}^{k_{n}} \varphi^{-1}\left(\frac{1}{i}\right) .
$$

For any $\tau>0$ and $T>0$, we thus have

$$
\mathbf{P}\left\{\sum_{i=1}^{k_{n}} X_{i}^{(n)}>T \tau\right\} \leqslant \mathbf{P}\left\{\left\|\left(X_{n}\right)\right\|_{\varphi, \infty}>\frac{T \tau}{\sum_{i=1}^{k_{n}} \varphi^{-1}(1 / i)}\right\} .
$$

Making use of (1.3) we obtain

$$
\mathbf{P}\left\{\sum_{i=1}^{k_{n}} X_{i}^{(n)}>T \tau\right\} \leqslant \frac{262 H_{\varphi}^{X}(n)}{\varphi\left(T \tau / \Delta_{\varphi}\left(k_{n}\right)\right)} .
$$

Consider the subsequence $n_{p}$ defined in (2.1), and set

$$
T=T_{p}=\varphi^{-1}\left(p^{\alpha} H_{\varphi}^{X}\left(n_{p}\right)\right) \Delta_{\varphi}\left(k_{n_{p}}\right),
$$

with $\alpha>1$. Set further $\sigma=1 / \varphi(\tau)$. From (3.19) and making use of (1.6) we obtain

$$
\mathbf{P}\left\{\sum_{i=1}^{k_{n_{p}}} X_{i}^{\left(n_{p}\right)}>T_{p} \tau\right\} \leqslant 262 \sigma p^{-\alpha} \text {. }
$$


By Borel Cantelli lemma we just have proved that, a.s. as $n \rightarrow \infty$,

$$
\sum_{i=1}^{k_{n_{p}}} X_{i}^{\left(n_{p}\right)} \leqslant \tau \varphi^{-1}\left(p^{\alpha} H_{\varphi}^{X}\left(n_{p}\right)\right) \Delta_{\varphi}\left(k_{n_{p}}\right) .
$$

We now complete the proof of Theorem 2.4 in the following way.

For $n \in\left[n_{p}, n_{p+1}\right]$, and with $\alpha^{\prime}>\alpha$, we have

$$
\begin{aligned}
\sum_{i=1}^{k_{n}} X_{i}^{(n)} & \leqslant \sum_{i=1}^{k_{n_{p+1}}} X_{i}^{\left(n_{p+1}\right)} \leqslant \tau \varphi^{-1}\left((p+1)^{\alpha^{\prime}} H_{\varphi}^{X}\left(n_{p+1}\right)\right) \Delta_{\varphi}\left(k_{n_{p+1}}\right) \\
& \leqslant \tau \varphi^{-1}\left((\log n)^{\alpha^{\prime}} H_{\varphi}^{X}\left(\left[n \alpha^{\prime}\right]\right)\right) \Delta_{\varphi}\left(k_{n} \alpha^{\prime}\right)
\end{aligned}
$$

where the second inequality holds a.s. for $n$ large enough. The upper bound in (3.21) enables to conclude the proof, by letting $\alpha^{\prime}$ arbitrarily close to $\alpha$.

\section{REFERENCES}

1. Bingham N., Goldie C., Teugels J. Regular Variation. - Cambridge: Cambridge Univ. Press, 1987.

2. Chow Y. S., Teicher H. Independence, Interchangeability, Martingales. Berlin-Heidelberg: Springer-Verlag, 1978.

3. Csörgö S., Mason D. The asymptotic distribution of sums of extreme values from a regularly varying distribution. - Ann. Probab., 1986, v. 14, № 3, p. 974-983.

4. Deheuvels P., Haeusler E., Mason D. Almost sure convergence of the Hill estimator. - Math. Proc. Cambridge Philos. Soc., 1988, v. 104, № 2, p. 371-381.

5. Deheuvels $P$., Mason $D$. The asymptotic behavior of sums of exponential extreme values. - Bull. Sci. Math., 1988, v. 112, p. 211-233.

6. Einmahl J., Haeusler E., Mason D. On the relationship between the almost sure stability of weighted empirical distributions and sums of order statistics. - Probab. Theory Relat. Fields, 1988, v. 79, № 1, p. 59-77.

7. Marcus $M$., Pisier $G$. Characterizations of almost surely continuous $p$-stable random Fourier series and strongly stationary processes. - Acta Math., 1984, v. 152, p. 245301.

8. Mason D. Laws of large numbers for sums of extreme values. - Ann. Probab., 1982, v. 10, NN 3, p. $754-764$.

9. Van Zuijlen $M$. C. A. Properties of the empirical distribution function for independent non-identically distributed random variables. - Ann. Probab., 1978, v. 6, № 2, p. $250-266$. 